\title{
Considerations for Outsourcing EU QPPV Services Taking into Account the Impact of Brexit
}

Author: Dr. Kosta Cvijovic

\section{1 | Background}

With the impending Brexit effect of European Union Qualified Persons for Pharmacovigilance (EU QPPV) no longer being able to reside in the UK, and several other issues that arose in discussions with potential and current clients regarding outsourcing pharmacovigilance (PV) services, Syneos Health believed it would be useful to conduct a survey among pharmaceutical and biotech companies we had interacted with as a service provider to determine the main considerations and challenges when outsourcing PV services in the current climate.

\section{2 | Methods}

The survey was purposefully designed to be short and possible to answer in 2 minutes with only 4 multiple choice

questions. In addition, the opportunity for in-depth conversation to further discuss the issues was offered to and taken by several respondents. The survey

respondents were companies that Syneos Health either provided PV services for or was involved with requests for proposals (RFP) in the past 24 months (2017-2019). The survey questions and answers were defined as follows:

1. What were you interested in outsourcing? - Full post-marketing PV including EU QPPV

Partial post-marketing PV tasks including EU QPPV

Partial post-marketing PV tasks excluding EU QPPV

2. How important to your outsourcing decision was the EU QPPV's non-UK location in light of Brexit?

-Important - Medium - Not at all
3. How important was pricing as a factor in your outsourcing decision as compared to established track record of quality?

Pricing more important than quality - Balance important

-Quality more important than pricing

4. What area of technological development was critical for your outsourcing decision? - Classic safety database product Cloud capabilities

- Al (artificial intelligence) capabilities

\section{3 | Results}

Thirty-one companies were contacted (16 biotech launching first product, 3 established generics, 12 established R\&D pharma with many marketed products) 12 provided feedback (38.7\%). The response rates to the questions are presented in the graph to the left. Most were looking to outsource partial PV functions, including the EU QPPV role. All respondents found the non-UK location of the QPPV to be critical. Regarding the balance of pricing vs. quality, a clear focus on pricing could be identified, though further analysis demonstrated that biotechs launching their first product and generics companies focused far and generics companies focused far more on pricing, while established R\&D pharmaceuticals were more interested in the quality track record of the service provider. The same correlation was also found in companies with no previous PV inspection experience (focus on pricing) and those with inspection experience (focus on quality). Finally, when it came to technology, it was clear that few companies were interested in a classic off-the-shelf database model and were particularly interested in the service provider's capabilities when it came to cloud-based and artificial intelligence (AI) solutions. In conversations, the reason for the focus on cloud was mainly the question of client access.

Survey Respondents

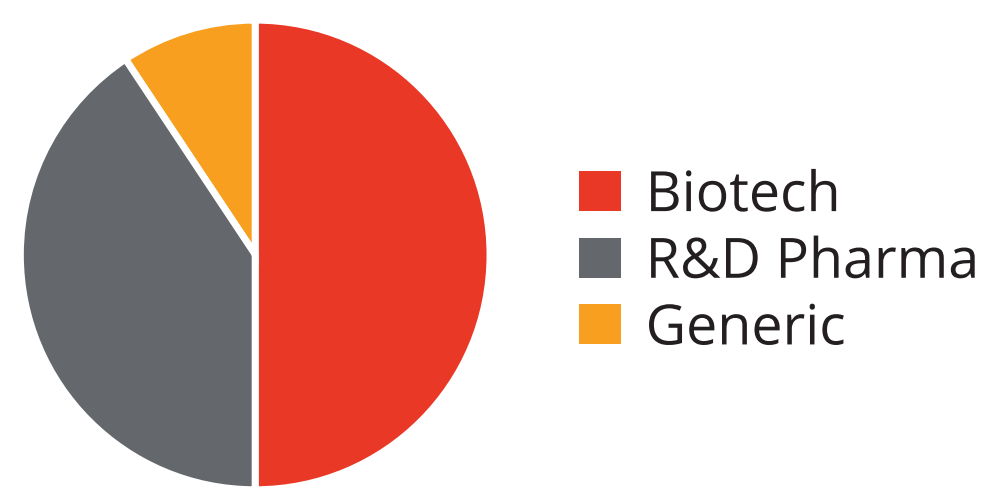

\section{4 | Conclusions}

It seems clear from the results that virtually no company is expecting the possibility of an EU QPPV to be able to remain in the UK after Brexit becomes a reality. This is a significant impact on the EU QPPV function

considering that the majority of EU QPPVs pre-Brexit were based in the UK. The data also points to PV being regarded primarily as a cost issue in the early stages of marketing, while quality is typically focused on in postinspection phases. Finally, digital capabilities were identified as a major consideration for outsourcing in PV. Further developments in this area, such as Al-based PV applications, will become more and more important for service providers and marketing authorization holders (MAHs) alike as development in this area continues. 